\title{
Orbit Representations from Linear mod 1 Transformations
}

\author{
Carlos CORREIA RAMOS ${ }^{\dagger}$, Nuno MARTINS ${ }^{\ddagger}$ and Paulo R. PINTO $\ddagger$ \\ $\dagger$ Centro de Investigação em Matemática e Aplicações, \\ R. Romão Ramalho, 59, 7000-671 Évora, Portugal \\ E-mail: ccr@uevora.pt \\ $\ddagger$ Department of Mathematics, CAMGSD, Instituto Superior Técnico, \\ Technical University of Lisbon, Av. Rovisco Pais, 1049-001 Lisboa, Portugal \\ E-mail:nmartins@math.ist.utl.pt,ppinto@math.ist.utl.pt
}

Received March 14, 2012, in final form May 09, 2012; Published online May 16, 2012

http://dx.doi.org/10.3842/SIGMA.2012.029

\begin{abstract}
We show that every point $x_{0} \in[0,1]$ carries a representation of a $C^{*}$-algebra that encodes the orbit structure of the linear mod 1 interval map $f_{\beta, \alpha}(x)=\beta x+\alpha$. Such $C^{*}$-algebra is generated by partial isometries arising from the subintervals of monotonicity of the underlying map $f_{\beta, \alpha}$. Then we prove that such representation is irreducible. Moreover two such of representations are unitarily equivalent if and only if the points belong to the same generalized orbit, for every $\alpha \in[0,1[$ and $\beta \geq 1$.
\end{abstract}

Key words: interval maps; symbolic dynamics; $C^{*}$-algebras; representations of algebras

2010 Mathematics Subject Classification: 46L55; 37B10; 46L05

\section{Introduction}

A famous class of representations of the Cuntz algebra $\mathcal{O}_{n}$ called permutative representations were studied and classified by Bratteli and Jorgensen in [2,3]. From the applications viewpoint, and besides its own right, applications of representation theory of Cuntz and Cuntz-Krieger algebras to wavelets, fractals, dynamical systems, see e.g. [2, 3, 13], and quantum field theory in [1] are particularly remarkable. For example, it is known that these representations of the Cuntz algebra serve as a computational tool for wavelets analysts, see [12]. This is clear because such a representation on a Hilbert space $H$ induces a subdivision of $H$ into orthogonal subspaces. Then the problem in wavelet theory is to build orthonormal bases in $L^{2}(\mathbb{R})$ from these data. Indeed this can be done [9] and these wavelet bases have the advantages over the earlier known basis constructions (one advantage is the efficiency of computation). This method has also been applied to the context of fractals that arise from affine iterated function systems [10]. Some of these results have been extended to the more general class of Cuntz-Krieger algebras, see [7, 13] and subshift $C^{*}$-algebras $[4,11,15]$ (whose underlying subshift is not necessarily of finite type) in $[5]$.

Symbolic dynamics is one of the main tools that we have used in $[5,7]$ to construct representations of Cuntz, Cuntz-Krieger and subshift $C^{*}$-algebras. The $C^{*}$-algebra is naturally associated to the given interval map and the Hilbert spaces naturally arise from the generalized orbits of the interval map. For a particular family of interval maps, we were able to recover Bratteli and Jorgensen permutative representations in [7] among the class of Markov maps (which underline the Cuntz-Krieger algebras of the transition matrix). We remark that while these Cuntz and Cuntz-Krieger algebras are naturally associated to the so-called Markov or periodic dynamical systems, the subshift $C^{*}$-algebras are ready to incorporate bigger classes of interval maps. 
The interval maps that we treat in [5] are unimodal maps (that have precisely two subintervals of monotonicity). Then the representations of the subshift $C^{*}$-algebra constructed in [5] are shown to coincide with the ones constructed in [7] from the Cuntz-Krieger algebra, provided the underlying dynamical system is periodic and therefore has a finite transition Markov Matrix. However, the proof of the irreducibility of the subshift $C^{*}$-algebras representations (for unimodal maps without a finite transition Markov matrix) rely on the structure of these unimodal interval maps where the $C^{*}$-algebra is generated by two partial isometries.

In this paper we construct representations (of a subshift $C^{*}$-algebra generated by $n$ partial isometries) from a family of interval maps and prove the irreducibility of these representations (avoiding the unimodal maps techniques used in [5]).

Namely, we yield and study representations of a certain $C^{*}$-algebra on the generalized orbit $\bigcup_{i \in \mathbb{Z}} f_{\beta, \alpha}^{j}\left(x_{0}\right)$ of every point $x_{0} \in[0,1]$ from the interval map $f_{\beta, \alpha}:[0,1] \rightarrow[0,1]$ defined by

$$
f_{\beta, \alpha}(x)=\beta x+\alpha \quad(\bmod 1) \quad \text { with } \beta \geq 1 \quad \text { and } \alpha \in[0,1[,
$$

by fixing the parameters $\alpha$ and $\beta$. The underlying $C^{*}$-algebra $\mathcal{O}_{\Lambda_{f_{\beta, \alpha}}}$ is generated by $n$ partial isometries where $n$ is the number of monotonicity subintervals of $f_{\beta, \alpha}$. See Fig. 1 for a graph of one such map.

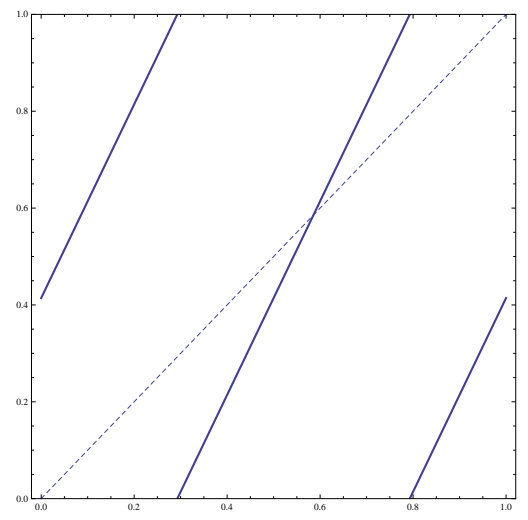

Figure 1. Graph of $f_{\beta, \alpha}$ with $\alpha=\sqrt{2}-1$ and $\beta=2(n=3)$.

We show that the representation is irreducible. Moreover the representations of the same algebra on the orbits of the two points $x_{0}$ and $y_{0}$ are unitarily equivalent if and only if the orbits coincide. If the parameters $\alpha$ and $\beta$ are so that the dynamical system $\left([0,1], f_{\beta, \alpha}\right)$ is periodic, the above results were obtained by $[6,7]$, where the relevant $C^{*}$-algebra is the Cuntz-Krieger $\mathcal{O}_{A_{f_{\beta, \alpha}}}$ and $A_{f_{\beta, \alpha}}$ is the underlying Markov transition 0-1 (finite) matrix of $f_{\beta, \alpha}$. A further particular case is obtained when $\beta=n$ is an integer and $\alpha=0$, in which case the $n \times n$ matrix $A_{f_{n, 0}}=\left(a_{i, j}\right)$ is full, $a_{i j}=1$ for all $i, j$, and thus recovering the Cuntz algebra $\mathcal{O}_{n}$ representations yielded in $[2,3]$ using wavelet theory framework.

Of course we may fairly easily prove that we do get representations of such $C^{*}$-algebras in the context of piecewise monotone maps, but the main concern is how to show irreducibility (and unitarily equivalence) of such the representations, thus giving a rich family of representations attached to interval maps. The periodic or non-periodic cases for which we have $n=2$ subintervals of monotonicity were carried out in [5]. We generalize here the construction of the representations for generic piecewise monotone interval maps and prove irreducibility and unitarily equivalence for the dynamical systems arising from equation (1) above - and obviously we look for values of the parameters $\alpha$ and $\beta$ for which we do not have a finite Markov transition matrix for $f_{\beta, \alpha}$ and thus we really get representations that cannot be recovered from $[5,6,7]$.

A more detailed description of the paper is as follows. In Section 2 we provide some background material first on the operator algebras setup and then in symbolic dynamics [16]. The 
main results are in Section 3. We consider a partition $\mathcal{I}$ of the interval $I=[0,1]$ into subintervals so that the restriction of $f_{\beta, \alpha}$ to each of these subintervals is monotone. Then for every $x_{0} \in I$ we explicitly define in equation (6) a linear operator on the Hilbert space $H_{x_{0}}$ that arises from the generalized orbit of $x_{0}$, for every such subinterval. The Hilbert space $H_{x_{0}}$ encodes the generalized orbit orbit $\left(x_{0}\right)$ of $x_{0}$ and in fact every $\xi \in \operatorname{orbit}\left(x_{0}\right)$ is regarded as a vector $|\xi\rangle$ in $H_{x_{0}}$ using Dirac's notation. Then we prove that these linear operators do satisfy the relation they ought to satisfy, leading to a representation $\rho_{x_{0}}: \mathcal{O}_{A_{f_{\beta, \alpha}}} \rightarrow B\left(H_{x_{0}}\right)$ of the $C^{*}$-algebra $\mathcal{O}_{A_{f_{\beta, \alpha}}}$, as in Proposition 2.

Then the main result of this paper is Theorem 1 where we show that representation $\rho_{x_{0}}$ of the underlying $C^{*}$-algebra $\mathcal{O}_{A_{f_{\beta, \alpha}}}$ is irreducible and that two such representations $\rho_{x_{0}}$ and $\rho_{y_{0}}$ are unitarily equivalent if and only if $y_{0}$ belongs to the generalized orbit of $x_{0}$. The new ingredient involved in the proof of Theorem 1 is the computation of the commutant $\mathcal{A}_{\beta, \alpha}^{\prime}$ of $C^{*}$-algebra $\mathcal{A}_{\beta, \alpha}$ (generated by the operators defined in equations (9) and (10)) in $B\left(H_{x_{0}}\right)$. Indeed, as soon as we prove that the commutant is trivial $\mathcal{A}_{\beta, \alpha}^{\prime}=\mathbb{C} 1$, we only have to show that $\mathcal{A}_{\beta, \alpha}^{\prime}$ contains $\rho_{x_{0}}\left(\mathcal{O}_{A_{f_{\beta, \alpha}}}\right)^{\prime}$ as in Proposition 3.

\section{Preliminaries}

In this section we provide some necessary background, starting with the operator algebras we obtain from dynamical systems. A representation of a $*$-algebra $\mathcal{A}$ on a complex Hilbert space $H$ is a $*$-homomorphism $\pi: \mathcal{A} \rightarrow B(H)$ into the *-algebra $B(H)$ of bounded linear operators on $H$. Usually representations are studied up to unitary equivalence. Two representations $\pi: \mathcal{A} \rightarrow B(H)$ and $\widetilde{\pi}: \mathcal{A} \rightarrow B(\widetilde{H})$ are (unitarily) equivalent if there is a unitary operator $U: H \rightarrow \widetilde{H}$ (i.e., $U$ is a surjective isometry) such that

$$
U \pi(a)=\tilde{\pi}(a) U \text { for every } a \in \mathcal{A},
$$

and in this case we write $\pi \sim \tilde{\pi}$. A representation $\pi: \mathcal{A} \rightarrow B(H)$ of some $*$-algebra is said to be irreducible if there is no non-trivial subspace of $H$ invariant with respect to all operators $\pi(a)$ with $a \in \mathcal{A}$. A well known result, see e.g. [17, Proposition 3.13], says that $\pi$ is irreducible if and only if

$$
x \in B(H): x \pi(a)=\pi(a) x \quad \text { for all } a \in \mathcal{A} \Longrightarrow x=\lambda \mathbf{1},
$$

for some complex number $\lambda$, where 1 denotes the identity of $B(H)$. By the very definition of commutant, $(2)$ can be restated as follows: $\pi(\mathcal{A})^{\prime}=\mathbb{C} \mathbf{1}$. We will be interested in some classes of $C^{*}$-algebras (= Banach $*$-algebras such that $\left\|a a^{*}\right\|=\|a\|^{2}$ holds for all $a$, see e.g. [17]). Besides, if we have a representation $\pi: A \rightarrow B(H)$ of a $C^{*}$-algebra $A$, then $\pi$ being a *homomorphism implies that $\|\pi(a)\| \leq\|a\|$ for all $a \in A$, thus $\pi$ is automatically continuous, see also e.g. [17, Secttion 1.5.7]).

\section{Subshift $C^{*}$-algebras}

Let $\Lambda \subseteq \Sigma^{\mathbb{N}}$ be a subshift with a finite alphabet $\Sigma=\{1, \ldots, n\}$. Exel [11] and Matsumoto [15] constructed $C^{*}$-algebras associated to $\Lambda$. Carlsen and Silvestrov [4] unified the two constructions that led them to a $C^{*}$-algebra $\mathcal{O}_{\Lambda}$, which is unital and generated partial isometries $\left\{t_{i}\right\}_{i \in \Sigma}$. Then the partial isometries that generate $\mathcal{O}_{\Lambda}$ obey the following relations:

$$
\sum t_{i} t_{i}^{*}=\mathbf{1}, \quad t_{\alpha}^{*} t_{\alpha} t_{\beta}=t_{\beta} t_{\alpha \beta}^{*} t_{\alpha \beta}, \quad t_{\alpha}^{*} t_{\alpha} t_{\beta}^{*} t_{\beta}=t_{\beta}^{*} t_{\beta} t_{\alpha}^{*} t_{\alpha},
$$

where $t_{\alpha}=t_{\alpha_{1}} \cdots t_{\alpha_{|\alpha|}}$ and $t_{\beta}=t_{\beta_{1}} \cdots t_{\beta_{|\beta|}}$ with $\alpha, \beta$ admissible words (if $\alpha=\left(\alpha_{1}, \ldots, \alpha_{k}\right.$ ) with $\alpha_{i} \in \Sigma$ we denote by $|\alpha|$ the length $k$ of $\alpha$ ). The algebra $\mathcal{O}_{\Lambda}$ is called the $C^{*}$-algebra associated 
to the subshift $\Lambda$ or subshift $C^{*}$-algebra. Important properties of the subshift $C^{*}$-algebra $\mathcal{O}_{\Lambda}$ (e.g. simplicity) are naturally inherited from properties of the subshift $\Lambda$. If $\Lambda$ is a subshift of finite type, then $\mathcal{O}_{\Lambda}$ is nothing but the well known Cuntz-Krieger algebra [15], where the Cuntz-Krieger algebra $\mathcal{O}_{A}$ associated to a 0-1 matrix $A=\left(a_{i j}\right)$ is the $C^{*}$-algebra [8] generated by (non-zero) partial isometries $s_{1}, \ldots, s_{n}$ satisfying:

$$
s_{i}^{*} s_{i}=\sum_{j=1}^{n} a_{i j} s_{j} s_{j}^{*} \quad(i=1, \ldots, n), \quad \sum_{i=1}^{n} s_{i} s_{i}^{*}=\mathbf{1},
$$

and the Cuntz algebra $\mathcal{O}_{n}$ is the Cuntz-Krieger algebra $\mathcal{O}_{A}$ with $A$ full $a_{i j}=1$ for all $1 \leq i, j \leq n$.

\subsection{Symbolic dynamics on piecewise monotone interval maps}

Let $f: I \rightarrow I$ be a piecewise monotone map of the interval $I$ into itself, that is, there is a minimal partition of open sub-intervals of $I, \mathcal{I}=\left\{I_{1}, \ldots, I_{n}\right\}$ such that $\overline{\bigcup_{j=1}^{n} I_{j}}=I$ and $f_{\mid I_{j}}$ is continuous monotone, for every $j=1, \ldots, n$. We define $f_{j}:=f_{\mid I_{j}}$. The inverse branches are denoted by $f_{j}^{-1}: f\left(I_{j}\right) \rightarrow I_{j}$. Let $\chi_{I_{i}}$ be the characteristic function on the interval $I_{i}$. The following are naturally satisfied

$$
f \circ f_{j}^{-1}(x)=\chi_{f\left(I_{j}\right)}(x) x, \quad f_{j}^{-1} \circ f_{\mid I_{j}}(x)=\chi_{I_{j}}(x) x .
$$

Let $\{1,2, \ldots, m\}$ be the alphabet associated to some partition $\left\{J_{1}, \ldots, J_{m}\right\}$ of open subintervals of $I$ so that $\overline{\bigcup_{j=1}^{m} J_{j}}=I$, not necessarily $\mathcal{I}$. The address map, is defined by

$$
\text { ad }: \bigcup_{j=1}^{m} J_{j} \rightarrow\{1,2, \ldots, m\}, \quad \operatorname{ad}(x)=i \quad \text { if } x \in J_{i} .
$$

We define

$$
\Omega_{f}:=\left\{x \in I: f^{k}(x) \in \bigcup_{j=1}^{m} J_{j} \text { for all } k=0,1, \ldots\right\} .
$$

Note that $\bar{\Omega}_{f}=I$. The itinerary map it $: \Omega_{f} \rightarrow\{1,2, \ldots, m\}^{\mathbb{N}}$ is defined by

$$
\operatorname{it}(x)=\operatorname{ad}(x) \operatorname{ad}(f(x)) \operatorname{ad}\left(f^{2}(x)\right) \cdots
$$

and let

$$
\Lambda_{f}=\operatorname{it}\left(\Omega_{f}\right)
$$

The space $\Lambda_{f}$ is invariant under the shift map $\sigma:\{1,2, \ldots, m\}^{\mathbb{N}} \rightarrow\{1,2, \ldots, m\}^{\mathbb{N}}$ defined by

$$
\sigma\left(i_{1} i_{2} \cdots\right)=\left(i_{2} i_{3} \cdots\right)
$$

and we have it $\circ f=\sigma \circ$ it. We will use $\sigma$ meaning in fact $\sigma_{\mid \Lambda_{f}}$. A sequence in $\{1,2, \ldots, m\}^{\mathbb{N}}$ is called admissible, with respect to $f$, if it occurs as an itinerary for some point $x$ in $I$, that is, if it belongs to $\Lambda_{f}$. An admissible word is a finite sub-sequence of some admissible sequence. The set of admissible words of size $k$ is denoted by $W_{k}=W_{k}(f)$. Given $i_{1} \cdots i_{k} \in W_{k}$, we define $I_{i_{1} \cdots i_{k}}$ as the set of points $x$ in $\Omega_{f}$ which satisfy

$$
\operatorname{ad}(x)=i_{1}, \ldots, \operatorname{ad}\left(f^{k}(x)\right)=i_{k} .
$$




\subsection{Linear mod 1 interval maps}

Now, let us consider the family of linear mod 1 transformations as in equation (1). In the sequel we will denote $f_{\beta, \alpha}$ by $f$. The behavior of the dynamical system $(I, f)$ is characterized by the sequences it $\left(f\left(c_{j}^{+}\right)\right)$and $\operatorname{it}\left(f\left(c_{j}^{-}\right)\right)$, for each discontinuity point $c_{j}$, see [14]. Let us consider the partition of monotonicity $\mathcal{I}=\left\{I_{1}, \ldots, I_{n}\right\}$ of $f$, with

$$
\begin{gathered}
\left.I_{1}=\right] 0,(1-\alpha) / \beta\left[, \ldots, I_{j}=\right](j-\alpha) / \beta,(j+1-\alpha) / \beta[, \ldots, \\
\left.\ldots, I_{n}=\right](n-1-\alpha) / \beta, 1[,
\end{gathered}
$$

which is the minimal partition of monotonicity for $f(n=[\beta]+1$ with $[\beta]$ being the integral part of $\beta$ and $\alpha>0$. For $\alpha=0: n=[\beta]$ if $\beta$ is an integer, and $n=[\beta]+1$ if $\beta \notin \mathbb{N}$ ). A characterization of the values of $\alpha$, for which there is a Markov partition, is partially given by the following:

Proposition 1. If $\operatorname{it}_{f}(0)=\left(\xi_{1}, \xi_{2}, \ldots, \xi_{l}, \ldots\right)$ and $\operatorname{it}_{f}(1)$ are periodic $\left(\right.$ with $\left.\xi_{l}=\xi_{1}\right)$ then

$$
\alpha=\frac{\xi_{l}+\xi_{l-1} \beta+\xi_{l-2} \beta^{2}+\cdots+\xi_{1} \beta^{l-1}}{1+\beta+\beta^{2}+\cdots+\beta^{l-1}} .
$$

In particular $\alpha \in \mathbb{Q}(\beta)$.

Proof. See [14, Proposition 2.6] for full details.

\section{Subshift algebras from linear mod 1 transformations}

As in [7], we consider the equivalence relation

$$
R_{f}=\left\{(x, y): f^{n}(x)=f^{m}(y) \text { for some } n, m \in \mathbb{N}_{0}\right\} .
$$

We write $x \sim y$ whenever $(x, y) \in R_{f}$. Consider the equivalence class $R_{f}(x)\left(=\bigcup_{j \in \mathbb{Z}} f^{j}(x)\right.$ also called the generalized orbit of $x)$ and set $H_{x}$ the Hilbert space $l^{2}\left(R_{f}(x)\right)$ with canonical orthonormal basis $\left\{|y\rangle: y \in R_{f}(x)\right\}$, in Dirac notation. Note that $H_{x}=H_{y}$ (are the same Hilbert spaces) whenever $x \sim y$. The inner product $(\cdot, \cdot)$ is given by

$$
\langle y \mid z\rangle=(|y\rangle,|z\rangle)=\delta_{y, z} .
$$

Let now $f$ be the linear mod 1 transformation defined in equation $(1)$ and $\mathcal{I}=\left\{I_{1}, \ldots, I_{n}\right\}$ be the partition of monotonicity as written down in equation (5). For every $i=1, \ldots, n$, let $f_{i}:=f_{\mid I_{i}}$ be the restriction of $f$ to the subinterval $I_{i}$ of the partition $\mathcal{I}$. For every $i \in\{1,2, \ldots, n\}$ let us define an operator $T_{i}$ on $H_{x}$ defined first on the orthonormal basis as follows:

$$
T_{i}|y\rangle=\chi_{f\left(I_{i}\right)}(y)\left|f_{i}^{-1}(y)\right\rangle
$$

and then extend it by linearity and continuity to $H_{x}$. Note that $\chi_{f\left(I_{i}\right)}(x)=1$ if and only if there is a pre-image of $x$ in $I_{i}$. The we have

$$
T_{i}^{*}|y\rangle=\chi_{I_{i}}(y)|f(y)\rangle .
$$

Indeed, on one hand

$$
\left(|y\rangle, T_{i}|z\rangle\right)=\left(|y\rangle, \chi_{f\left(I_{i}\right)}\left|f_{i}^{-1}(z)\right\rangle\right)
$$

and on the other hand we have

$$
\left(T_{i}^{*}|y\rangle,|z\rangle\right)=\left(\chi_{I_{i}}(y)|f(y)\rangle,|z\rangle\right)=\chi_{I_{i}}(y) \delta_{f(y), z} .
$$

So since $\chi_{f\left(I_{i}\right)} \mid f_{i}^{-1}(z)=\chi_{I_{i}}(y) \delta_{f(y), z}$ we have shown that the adjoint of $T_{i}$ is given by equation (7). We further remark that $T_{i}$ is a partial isometry: namely, $T_{i}$ is an isometry on its restriction to $\operatorname{span}\left\{|y\rangle: y \in f\left(I_{i}\right)\right\} \cap H_{x}$ and vanishes in the remaining part of $H_{x}$. 
Lemma 1. The operators $T_{i}$ satisfy the relations

$$
\sum_{i=1}^{n} T_{i} T_{i}^{*}=\mathbf{1}, \quad T_{\mu}^{*} T_{\mu} T_{\nu}=T_{\nu} T_{\mu \nu}^{*} T_{\mu \nu} \quad \text { and } \quad T_{\mu}^{*} T_{\mu} T_{\nu}^{*} T_{\nu}=T_{\nu}^{*} T_{\nu} T_{\mu}^{*} T_{\mu}
$$

for $\mu, \nu$ given admissible words.

Proof. Consider $T_{i} T_{i}^{*}$ acting on a vector $|y\rangle$ of the canonical basis of $H_{x}$,

$$
T_{i} T_{i}^{*}|y\rangle=\chi_{I_{i}}(y) T_{i}|f(y)\rangle=\chi_{I_{i}}(y) \chi_{f\left(I_{i}\right)}(f(y))\left|f_{i}^{-1} \circ f(y)\right\rangle=\chi_{I_{i}}(y)|y\rangle,
$$

since $\chi_{I_{i}}(y) \chi_{f\left(I_{i}\right)}(f(y))=\chi_{I_{i}}(y)$. Then

$$
\left(T_{1} T_{1}^{*}+\cdots+T_{n} T_{n}^{*}\right)|y\rangle=\left(\chi_{I_{1}}(y)+\cdots+\chi_{I_{n}}(y)\right)|y\rangle=|y\rangle .
$$

Now, consider $T_{\mu}^{*} T_{\mu} T_{\nu}$ acting on a vector $|y\rangle$ of the canonical basis for some $\mu=\mu_{1} \cdots \mu_{k}$, $\nu=\nu_{1} \cdots \nu_{r}$ admissible words,

$$
\begin{aligned}
T_{\mu}^{*} T_{\mu} T_{\nu}|y\rangle & =T_{\mu}^{*} T_{\mu} \chi_{f^{r}\left(I_{\nu}\right)}(y)\left|f_{\nu_{1}}^{-1} \circ \cdots \circ f_{\nu_{r}}^{-1}(y)\right\rangle \\
& =T_{\mu}^{*} \chi_{f^{k+r}\left(I_{\mu \nu}\right)}(y)\left|f_{\mu_{1}}^{-1} \circ \cdots \circ f_{\mu_{k}}^{-1} \circ f_{\nu_{1}}^{-1} \circ \cdots \circ f_{\nu_{r}}^{-1}(y)\right\rangle \\
& =\chi_{f^{k+r}\left(I_{\mu \nu}\right)}(y)\left|f_{\nu_{1}}^{-1} \circ \cdots \circ f_{\nu_{r}}^{-1}(y)\right\rangle .
\end{aligned}
$$

On the other hand

$$
\begin{aligned}
T_{\nu} T_{\mu \nu}^{*} T_{\mu \nu}|y\rangle & =T_{\nu} T_{\mu \nu}^{*} \chi_{f^{k+r}\left(I_{\mu \nu}\right)}(y)\left|f_{\mu_{1}}^{-1} \circ \cdots \circ f_{\mu_{k}}^{-1} \circ f_{\nu_{1}}^{-1} \circ \cdots \circ f_{\nu_{r}}^{-1}(y)\right\rangle \\
& =T_{\nu} \chi_{f^{k+r}\left(I_{\mu \nu}\right)}(y)|y\rangle=\chi_{f^{k+r}\left(I_{\mu \nu}\right)}(y)\left|f_{\nu_{1}}^{-1} \circ \cdots \circ f_{\nu_{r}}^{-1}(y)\right\rangle .
\end{aligned}
$$

Finally since $T_{\mu}^{*} T_{\mu}|y\rangle=\chi_{f^{k}\left(I_{\mu}\right)}(y)|y\rangle$ and $T_{\nu}^{*} T_{\nu}|y\rangle=\chi_{f^{r}\left(I_{\nu}\right)}(y)|y\rangle$ for admissible words $\mu, \nu$, we easily conclude that $T_{\mu}^{*} T_{\mu} T_{\nu}^{*} T_{\nu}=T_{\nu}^{*} T_{\nu} T_{\mu}^{*} T_{\mu}$.

As an immediate consequence of Lemma 1 (and above equation (8)) we obtain the following.

Proposition 2. Let $\mathcal{O}_{\Lambda_{f}}$ be the subshift algebra associated to the subshift $\Lambda_{f}$ as defined in (4) above, then $\rho_{x}: \mathcal{O}_{\Lambda_{f}} \rightarrow B\left(H_{x}\right)$ defined by $t_{i} \rightarrow T_{i}$ is a representation of $\mathcal{O}_{\Lambda_{f}}$.

We remark here that $T_{i}^{*} T_{i}=\mathbf{1}$ for all $i=2, \ldots, n-1$ and $T_{1}^{*} T_{1}=\mathbf{1}$ if and only if $\alpha=0$. Besides $T_{n}^{*} T_{n}=\mathbf{1}$ if and only if $\beta=n \in \mathbb{N}$. Therefore $\rho_{x}$ is a representation of a Cuntz algebra if and only if $\alpha=0$ and $\beta$ is a positive integer. In this case, the interval map $f$ is a Markov map and moreover the partition with the monotonicity intervals, as in (5), reduces to

$$
\left.I_{1}=\right] 0,1 / n\left[, \quad I_{2}=\right] 1 / n, 2 / n\left[, \quad \ldots, \quad I_{j}=\right](j-1) / n, j / n\left[, \quad \ldots, \quad I_{n}=\right](n-1) / n, 1[
$$

and coincides with the (minimal) Markov partition [7]. From the viewpoint of interval maps, these Cuntz algebra $\mathcal{O}_{n}$ representations were treated in [7, Remark 2.9].

We remark that if $f_{\beta, \alpha}$ is a linear mod 1 map with $\alpha \notin \mathbb{Q}$ and $\beta=1$ then $f$ is not a Markov map by Proposition 1 and thus the representation $\rho_{x}$ of Proposition 2 is never a representation of a Cuntz-Krieger algebra. 


\subsection{Irreducibility of the representations}

For the linear mod 1 transformation map $f$ and the linear operators $T_{1}, \ldots, T_{n} \in B\left(H_{x}\right)$ defined in equation (6), we may consider the following operator

$$
V=T_{1}^{*}+\cdots+T_{n}^{*}
$$

which satisfies $V|y\rangle=|f(y)\rangle$ on every vector basis $|y\rangle$. In general $V$ is not unitary (unless $\beta=1$ so that $f$ becomes an invertible function). Let $U$ be the diagonal operator

$$
U|y\rangle=e^{2 \pi i y}|y\rangle
$$

which is an unitary operator, with $U^{*}|y\rangle=e^{-2 \pi i y}|y\rangle$. In order to emphasize that $U, V \in B\left(H_{x}\right)$, we write $U_{x}$ and $V_{x}$ for the above operators $U$ and $V$, respectively.

For a self-adjoint set of operators $\mathcal{S} \subseteq B(H)$ on some Hilbert space $H$, containing the identity $\mathbf{1}$, the von Neumann algebra generated by $\mathcal{S}$ equals the double commutant $\mathcal{S}^{\prime \prime}$, which in turn is also equal to the closure of $\mathcal{S}$ under the strong operator topology (this is the famous bicommutant von Neumann theorem e.g. the textbook [17]). We note that $\mathcal{S}^{\prime}=\{t \in B(H)$ : $t s=s t$, for all $s \in \mathcal{S}\}$ and $\mathcal{S}^{\prime \prime}=\left(\mathcal{S}^{\prime}\right)^{\prime}$ and $\mathcal{S}^{\prime \prime \prime}=\mathcal{S}^{\prime}$. Also $s_{i}$ converges to $s$ in the strong operator topology if $\left\|\left(s_{i}-s\right) \xi\right\| \rightarrow 0$ for every vector $\xi \in H$.

Let $\mathcal{A}_{\beta, \alpha}=C^{*}(U, V)$ be the $C^{*}$-subalgebra of $B\left(H_{x}\right)$ generated by $U$ and $V$, and consider the representation $\rho_{x}$ from Proposition 2.

Proposition 3. We have $\mathcal{A}_{\beta, \alpha} \subseteq \rho_{x}\left(\mathcal{O}_{\Lambda_{f}}\right)^{\prime \prime}$.

Proof. By definition of the $C^{*}$-algebra $\mathcal{A}_{\beta, \alpha}$, we only need to prove that $U, V$ belong to $\rho_{x}\left(\mathcal{O}_{\Lambda_{f}}\right)^{\prime \prime}$. It is clear that $V \in \rho_{x}\left(\mathcal{O}_{\Lambda_{f}}\right) \subseteq \rho_{x}\left(\mathcal{O}_{\Lambda_{f}}\right)^{\prime \prime}$. We now show that $U \in \rho_{x}\left(\mathcal{O}_{\Lambda_{f}}\right)^{\prime \prime}$. For each $\mu \in W_{k}$, let $m(\mu)$ be some point in $I_{\mu} \cap R_{f}(x)$. Note that if we have it $(y)=\left(\alpha_{j}\right)_{j=1}^{\infty}$, for some point $y \in R_{f}(x)$ then $\lim _{j \rightarrow \infty} m\left(\alpha_{1} \cdots \alpha_{j}\right)=y$, and the limit is independent on the choice of $m\left(\alpha_{1} \cdots \alpha_{j}\right) \in I_{\alpha_{1} \cdots \alpha_{j}}$, since for each $j \in \mathbb{N}$ there is $r>j$ so that $I_{\alpha_{1} \cdots \alpha_{j}} \supset I_{\alpha_{1} \cdots \alpha_{r}}$. Let $M_{k}=\sum_{\mu \in W_{k}} e^{2 \pi i m(\mu)} T_{\mu} T_{\mu}^{*}$. We can see that $\lim _{k \rightarrow \infty} M_{k}=U$ in the strong topology, since $\lim _{k \rightarrow \infty}\left\|M_{k} v-U v\right\|=0$, for every $v \in H_{x}$. Therefore, $U$ is in the von Neumann algebra generated by the operators $T_{1}, \ldots, T_{n}$.

Lemma 2. Let $x \in I$ and $f$ be the linear mod 1 transformation (1) with fixed $\alpha$ and $\beta$. Let $Q \in B\left(H_{x}\right)$ be an operator commuting with both $U$ and $V$, then $Q=\lambda I$ for some $\lambda \in \mathbb{C}$.

Proof. First of all we remark that the

$$
e^{2 \pi i z} \text { are all distinct, }
$$

with $z \in R_{f}(x)$, since $z \in[0,1]$. Let $Q \in \mathcal{B}\left(H_{x}\right)$ commuting with both $U$ and $V$. For each $z \in R_{f}(x)$ let $\mu_{z}:=e^{2 \pi i z}$. By definition $U|z\rangle=\mu_{z}|z\rangle$, i.e., every $\mu_{z}$ is an eigenvalue of $U$. We easily get

$$
U Q|z\rangle=\mu_{z} Q|z\rangle
$$

by applying the definitions and the fact that $U$ and $Q$ commute. For every $z \in R_{f}(x)$, set $\xi_{z}=Q|z\rangle$. Then we can write equation (12) as follows: $U \xi_{z}=\mu_{z} \xi_{z}$. Since $\left\{|z\rangle, z \in R_{f}(x)\right\}$ is an o.n. basis of $H_{x}$, there are constants $c_{w}$ with $w \in R_{f}(x)$ such that $\xi_{z}=\sum c_{w}|w\rangle$. Since

$$
U \xi_{z}=\sum_{w \in R_{f}(x)} \mu_{w} c_{w}|w\rangle
$$


and

$$
\mu_{z} \xi_{z}=\sum_{w \in R_{f}(x)} \mu_{z} c_{w}|w\rangle
$$

we conclude that $c_{w}=0$ for all $w \neq z$, because the $\mu_{w}$ 's are all distinct by (11) and $\{|w\rangle\}$ is an o.n. basis of $H_{x}$. It follows that $\xi_{z}=c_{z}|z\rangle$ or equivalently $Q|z\rangle=c_{z}|z\rangle$ for some $c_{z} \in \mathbb{C}$. But $V|z\rangle=|f(z)\rangle$, so $V Q=Q V$ gives $c_{z}=c_{f(z)}$. Therefore $Q$ is a multiple of the identity operator.

Theorem 1. The representation $\rho_{x}$ of the subshift $C^{*}$-algebra $\mathcal{O}_{\Lambda_{f}}$ as in Proposition 2 is irreducible. Moreover $\rho_{x} \sim \rho_{y}$ if and only if $x \sim y$.

Proof. To prove that $\rho_{x}$ is irreducible we prove that $\rho_{x}\left(\mathcal{O}_{\Lambda_{f}}\right)^{\prime}=\mathbb{C} I$. First note that from Proposition 3 we have $C^{*}(U, V) \subseteq \rho_{x}\left(\mathcal{O}_{\Lambda_{f}}\right)^{\prime \prime}$ and so taking commutant and using von Neumann bicommutant theorem, we conclude that $\rho_{x}\left(\mathcal{O}_{\Lambda_{f}}\right)^{\prime} \subseteq C^{*}(U, V)^{\prime}$.

Now let $Q \in \rho_{x}\left(\mathcal{O}_{\Lambda_{f}}\right)^{\prime}$. So we can conclude that $Q \in C^{*}(U, V)^{\prime}$ and thus $Q$ commutes with both $U$ and $V$. By Lemma 2 we conclude that $Q=\lambda I$ for some $\lambda \in \mathbb{C}$. Therefore $\rho_{x}\left(\mathcal{O}_{\Lambda_{f}}\right)^{\prime}=\mathbb{C} I$ and so $\rho_{x}$ is an irreducible representation of $\mathcal{O}_{\Lambda_{f}}$.

It is clear that if $R_{f}(x)=R_{f}(y)$, then $\rho_{x}$ and $\rho_{y}$ are unitarily equivalent. Notice that $U_{x}$ and $U_{y}$ have the same eigenvalues if and only if $x \sim y$. Hence $\rho_{x}$ and $\rho_{y}$ can be unitarily equivalent only when $x \sim y$.

Remark 1. If $\beta=n$ is an integer and $\alpha=0$ then the partition of $I$ into monotonicity subintervals of $f_{n, 0}$ is a Markov partition, the subshift $\Lambda_{f_{n, 0}}$ is the full shift and the underlying $C^{*}$-algebra is the Cuntz algebra $\mathcal{O}_{n}$. Furthermore we recover in Theorem 1 our previous result obtained in [7].

\section{Acknowledgment}

First author acknowledges CIMA-UE for financial support. The other authors were partially supported by the Fundação para a Ciência e a Tecnologia through the Program POCI 2010/FEDER.

\section{References}

[1] Abe M., Kawamura K., Recursive fermion system in Cuntz algebra. I. Embeddings of fermion algebra into Cuntz algebra, Comm. Math. Phys. 228 (2002), 85-101, math-ph/0110003.

[2] Bratteli O., Jorgensen P.E.T., Iterated function systems and permutation representations of the Cuntz algebra, Mem. Amer. Math. Soc. 139 (1999), no. 663, 89 pages, funct-an/9612002.

[3] Bratteli O., Jorgensen P.E.T., Ostrovs'kyı̌ V., Representation theory and numerical AF-invariants. The representations and centralizers of certain states on $\mathcal{O}_{d}$, Mem. Amer. Math. Soc. 168 (2004), no. 797, 178 pages, math.OA/9907036.

[4] Carlsen T.M., Silvestrov S., $C^{*}$-crossed products and shift spaces, Expo. Math. 25 (2007), 275-307, math.OA/0512488.

[5] Correia Ramos C., Martins N., Pinto P.R., On $C^{*}$-algebras from interval maps, Complex Anal. Oper. Theory, to appear.

[6] Correia Ramos C., Martins N., Pinto P.R., Orbit representations and circle maps, in Operator Algebras, Operator Theory and Applications, Oper. Theory Adv. Appl., Vol. 181, Birkhäuser Verlag, Basel, 2008, $417-427$.

[7] Correia Ramos C., Martins N., Pinto P.R., Sousa Ramos J., Cuntz-Krieger algebras representations from orbits of interval maps, J. Math. Anal. Appl. 341 (2008), 825-833. 
[8] Cuntz J., Krieger W., A class of $C^{*}$-algebras and topological Markov chains, Invent. Math. 56 (1980), 251-268.

[9] Daubechies I., Ten lectures on wavelets, CBMS-NSF Regional Conference Series in Applied Mathematics, Vol. 61, Society for Industrial and Applied Mathematics (SIAM), Philadelphia, PA, 1992.

[10] Dutkay D.E., Jorgensen P.E.T., Wavelet constructions in non-linear dynamics, Electron. Res. Announc. Amer. Math. Soc. 11 (2005), 21-33, math.DS/0501145.

[11] Exel R., A new look at the crossed-product of a $C^{*}$-algebra by an endomorphism, Ergodic Theory Dynam. Systems 23 (2003), 1733-1750, math.OA/0012084.

[12] Jorgensen P.E.T., Certain representations of the Cuntz relations, and a question on wavelets decompositions, in Operator Theory, Operator Algebras, and Applications, Contemp. Math., Vol. 414, Amer. Math. Soc., Providence, RI, 2006, 165-188, math.CA/0405372.

[13] Marcolli M., Paolucci A.M., Cuntz-Krieger algebras and wavelets on fractals, Complex Anal. Oper. Theory 5 (2011), 41-81, arXiv:0908.0596.

[14] Martins N., Sousa Ramos J., Cuntz-Krieger algebras arising from linear mod one transformations, in Differential Equations and Dynamical Systems (Lisbon, 2000), Fields Inst. Commun., Vol. 31, Amer. Math. Soc., Providence, RI, 2002, 265-273.

[15] Matsumoto K., On $C^{*}$-algebras associated with subshifts, Internat. J. Math. 8 (1997), 357-374.

[16] Milnor J., Thurston W., On iterated maps of the interval, in Dynamical Systems (College Park, MD, 19861987), Lecture Notes in Math., Vol. 1342, Springer, Berlin, 1988, 465-563.

[17] Pedersen G.K., $C^{*}$-algebras and their automorphism groups, London Mathematical Society Monographs, Vol. 14, Academic Press Inc., London, 1979. 